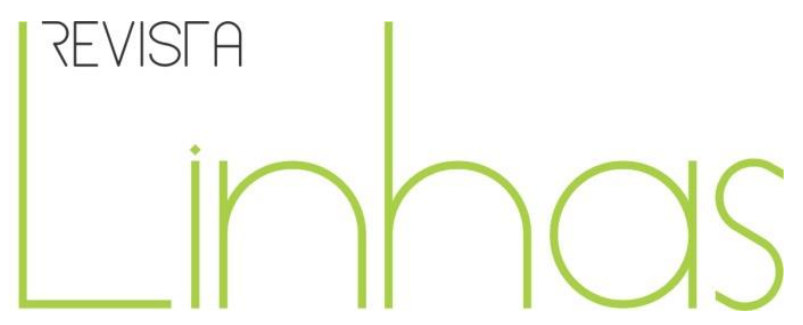

\title{
Tecnologia assistiva no Atendimento Educacional Especializado (AEE) de estudantes com deficiência
}

\begin{abstract}
Resumo
No âmbito da inclusão escolar, a política educacional brasileira orienta que a educação de estudantes com deficiência seja realizada no ensino comum. Nesse sentido, torna-se necessário o favorecimento de ambientes acessíveis e com acessibilidade que possibilitem o pleno desenvolvimento dessa população no processo de escolarização, quando o uso de tecnologias, sobretudo da Tecnologia Assistiva (TA) se torna ainda mais evidente. Para tanto, entre suas ações, as escolas comuns devem ofertar o Atendimento Educacional Especializado (AEE) que vem sendo priorizado por parte do governo federal, nos últimos anos, em Salas de Recursos Multifuncionais (SRM). O AEE é definido pela Política Nacional de Educação Especial na Perspectiva da Educação Inclusiva como aquele que tem por finalidade identificar, elaborar e organizar recursos pedagógicos e de acessibilidade que eliminem as barreiras para que os estudantes possam participar de todas as atividades, considerando suas necessidades específicas. Este texto tem como objetivo abordar o uso da TA no AEE em SRM para estudantes com deficiência, visando subsidiar um estudo em desenvolvimento sobre a utilização dessa tecnologia na oferta do AEE para essa população em dois municípios do interior do estado de Mato Grosso do Sul (MS).
\end{abstract}

Palavras-chave: Atendimento Educacional Especializado; Inclusão Escolar; Tecnologia Assistiva.
Nesdete Mesquita Corrêa

Universidade Federal do Mato

Grosso do Sul - UFMS - MS/Brasil nesdetemesquita@gmail.com

\section{Ana Paula Neves Rodrigues}

Secretaria Municipal de Educação

de Corumbá - MS/Brasil apnevesrodrigues@gmail.com

\footnotetext{
Para citar este artigo:

CORRÊA, Nesdete Mesquita; RODRIGUES, Ana Paula Neves. Tecnologia assistiva no Atendimento Educacional Especializado (AEE) de estudantes com deficiência. Revista Linhas. Florianópolis, v. 17, n. 35, p. 87-101, set./dez. 2016.
} 


\title{
Assistive technology in the Specialized Educational Service (SES) for students with disabilities
}

\begin{abstract}
In the school inclusion scope, the Brazilian educational policy directs that the education of students with disabilities be fulfilled in common education. This way, it is necessary favoring accessible environments and accessibility to enable the full development of this population in the schooling process, when the use of technology, especially of assistive technology (AT), becomes even more evident. Therefore, among their actions the regular schools must offer the Specialized Educational Service (SES) which has been prioritized by the federal government, in the last years, in Multifunctional Resource Classrooms (MRC). The SES is defined by the National Policy of Special Education in the Inclusive Education perspective as one that aims to identify, elaborate and organize educational and accessibility features that eliminate barriers so that students can participate in all activities, considering their specific needs. This text's goal is to address the use of AT in the SES in MRC for students with disabilities, in order to support a study in development on the use of this technology in the SES offer for this population in two cities in the state of Mato Grosso do Sul (MS).
\end{abstract}

Keywords: Specialized Educational Service; School Inclusion; Assistive Technology. 


\section{Introdução}

Nas duas últimas décadas, no Brasil, a inclusão escolar é assegurada por políticas educacionais, por leis e por outros documentos que respaldam o acesso e a permanência de estudantes com deficiência no ensino comum.

No contexto educacional brasileiro, os princípios da inclusão escolar se desenharam a partir dos anos 1990, no âmbito do movimento da escola para todos, quando o movimento de inclusão escolar se amplia e a inclusão de estudantes com deficiência em escolas comuns passa a ser defendida. É relevante considerar a afirmação de Kassar (2012) sobre essa questão, quando a autora assinala que para a educação especial, a inclusão desses estudantes surgiu como meio de superação das condições de segregação e de exclusão social a que foram historicamente submetidos.

Cumpre destacar que, em nosso país, a trajetória da oferta do Atendimento Educacional Especializado a essa população vem se constituindo sob a marca da expansão da oferta dos atendimentos, permeada por mudanças, exigindo a ação do Estado para atender à demanda da população no sentido de formular e implementar políticas públicas para esse fim (CORRÊA, 2012), as quais vêm sendo materializadas mediante diversos dispositivos normativos e orientadores.

Com a LDB n 9.394/1996, a educação especial é definida como modalidade de ensino, indicando a prerrogativa do apoio à inclusão de estudantes com deficiência no ensino comum por meio da organização e oferta do Atendimento Educacional Especializado (AEE). A LDB, no artigo 59, inciso I, evidencia que os sistemas de ensino assegurarão aos estudantes com deficiência, transtornos globais do desenvolvimento e altas habilidades ou superdotação: “I - currículos, métodos, técnicas, recursos educativos e organização específicos, para atender às suas necessidades.”

Assim, a LDB propõe uma nova abordagem educacional a ser oferecida a essa população, tornando-se imprescindível que o (a) professor (a) observe e identifique a existência de barreiras que limitem ou impeçam o estudante de participar ativamente do processo escolar.

Desse modo, para o atendimento do público-alvo da educação especial, torna-se necessário o favorecimento de ambientes acessíveis e com acessibilidade que 
possibilitem o pleno desenvolvimento dessa população no processo de escolarização. Para efeito dessas orientações, os termos acessibilidade e acessível são definidos pela Associação Brasileira de Normas Técnicas (ABNT, 2004, p. 2) como:

3.1 acessibilidade: Possibilidade e condição de alcance, percepção e entendimento para a utilização com segurança e autonomia de edificações, espaço, mobiliário, equipamento urbano e elementos.

3.2 acessível: Espaço, edificação, mobiliário, equipamento urbano ou elemento que possa ser alcançado, acionado, utilizado e vivenciado por qualquer pessoa, inclusive aquelas com mobilidade reduzida. O termo acessível implica tanto acessibilidade física como de comunicação. (grifos nossos)

$\mathrm{Na}$ atualidade, é inegável o avanço no uso das tecnologias, sobretudo no campo educacional. Em face à perspectiva da inclusão escolar, o uso da Tecnologia Assistiva se torna ainda mais evidente. A Tecnologia Assistiva (TA) é definida segundo o Comitê de Ajudas Técnicas (CAT), instituído no Brasil pela Portaria $n^{\circ}$ 142, de 16 de novembro de 2006, como:

[...] uma área do conhecimento, de característica interdisciplinar, que engloba produtos, recursos, metodologias, estratégias, práticas e serviços que objetivam promover a funcionalidade, relacionada à atividade e participação de pessoas com deficiência, incapacidades ou mobilidade reduzida, visando sua autonomia, independência, qualidade de vida e inclusão social. (BRASIL, 2007a)

Alguns estudos desenvolvidos sobre o tema (PELOSI, 2008; MIRANDA; GALVÃO FILHO, 2012; BERSCH, 2013, entre outros) elucidam a inclusão de estudantes com algum tipo de deficiência no ensino comum e sua escolarização possibilitada por meio da Tecnologia Assistiva. Nesse processo, ocorre a oferta do AEE aos estudantes que dele necessitam.

O presente estudo, ao se referir aos recursos de acessibilidade, evidencia a Tecnologia Assistiva (TA) aplicada à educação sob a forma de AEE (BRASIL, 2010a, p. 8) em Salas de Recursos Multifuncionais (SRM). 
Diante do exposto, este texto tem por objetivo abordar o uso da TA no AEE em SRM para estudantes com deficiência, visando subsidiar um estudo em desenvolvimento sobre a utilização dessa tecnologia na oferta do AEE para essa população nos municípios de Corumbá e Ladário, no interior do estado de Mato Grosso do Sul (MS).

Cabe registrar que os respectivos municípios apresentam os seguintes indicadores de escolas ${ }^{1}$, de matrículas e de salas de recursos multifuncionais: Corumbá possui dezesseis escolas municipais na área urbana com o ensino fundamental, das quais seis com Sala de Recursos Multifuncionais para a oferta do AEE. Segundo os dados do Inep, no ano de $2014^{2}$, os números de matrículas finais na Educação Básica nesse município se totalizaram em 15.015, com 218 matrículas de incluídos ${ }^{3}$. O município de Ladário possui sete escolas municipais na área urbana com o ensino fundamental, sendo quatro delas com Sala de Recursos Multifuncionais para a oferta do AEE. Em 2014, com um total de 3.515 matrículas, sendo 47 dessas matrículas de incluídos.

\section{Atendimento Educacional Especializado de estudantes com deficiência: Tecnologia Assistiva e Salas de Recursos Multifuncionais}

Para iniciar a exposição sobre o tema é importante destacar que em tempos de políticas de inclusão escolar, a oferta de Atendimento Educacional Especializado tem se vislumbrado como uma das estratégias viabilizadoras de condições de acesso, permanência e progressão escolar com sucesso de estudantes com deficiência no ensino comum.

Tal assertiva se confirma por meio da Política Nacional de Educação Especial na Perspectiva da Educação Inclusiva (PNEEI, 2008) que define que o AEE “[...] tem como função identificar, elaborar e organizar recursos pedagógicos e de acessibilidade que eliminem as barreiras para a plena participação dos alunos, considerando suas necessidades específicas" (BRASIL, 2008, p. 10).

\footnotetext{
${ }^{1}$ Os dados relativos ao número de escolas com sala de Atendimento Educacional Especializado foram obtidos do censo do sistema on-line: QEdu, de domínio público. Disponível em: <http://www.qedu.org.br/>. Acesso em: 30 jul. 2015.

2 Fonte: Inep. Disponível em: <http://portal.inep.gov.br/basica-censo-escolar-matricula>. Acesso em: 27 out.2015.

${ }^{3}$ Termo adotado pelo Inep/MEC para se referir à matrícula, no ensino comum, do público-alvo da educação especial.
} 
Em 2007, com o lançamento do Plano de Desenvolvimento da Educação (PDE), o MEC instituiu por meio da Portaria $\mathrm{n}^{\circ}$ 13, de 24 de abril de 2007 (BRASIL, 2007b, art. $1^{\circ}$ ), o Programa de Implantação de Salas de Recursos Multifuncionais, com o objetivo de "[...] apoiar os sistemas públicos de ensino na organização e oferta do atendimento educacional especializado e contribuir para o fortalecimento do processo de inclusão educacional nas classes comuns de ensino”. Neste documento (BRASIL, 2007b, Parágrafo único, art. $1^{\circ}$ ), as Salas de Recursos Multifuncionais foram definidas como "[...] espaço organizado com equipamentos de informática, ajudas técnicas, materiais pedagógicos e mobiliários adaptados, para atendimento às necessidades educacionais especiais dos alunos".

No ano em referência e como parte integrante das ações do Plano de Ações Articuladas (PAR), plano executor do PDE, visando garantir o acesso e permanência no ensino regular e o atendimento às necessidades educacionais especiais dos estudantes público-alvo da educação especial, o "Programa de Implantação de Salas de Recursos Multifuncionais" foi publicado pelo Edital $n^{\circ} 01$, de 26 de abril de 2007, com o objetivo de:

Apoiar os sistemas de ensino na organização e oferta do atendimento educacional especializado, por meio da implantação de salas de recursos multifuncionais nas escolas de educação básica da rede pública, fortalecendo o processo de inclusão nas classes comuns de ensino regular. (BRASIL, 2007C)

O programa visa disponibilizar, aos sistemas públicos de ensino, equipamentos de informática, mobiliários, materiais pedagógicos e de acessibilidade, com vistas a apoiar a ampliação da oferta do AEE. Entre os recursos se encontram os de Tecnologia Assistiva (TA).

Bersch (2013) afirma que a TA, ao ser inserida por meio das Salas de Recursos Multifuncionais (SRM), atribui ao professor do AEE a tarefa de reconhecer as necessidades de recursos pedagógicos e de Tecnologia Assistiva que melhor atendem o estudante na escola comum. 
Neres e Corrêa (2015) evidenciam o uso da Tecnologia Assistiva (TA) como recurso de apoio ao trabalho didático envolvendo estudantes com deficiência e englobam metodologias, estratégias, práticas e serviços que possam subsidiar a acessibilidade ou o processo pedagógico, visando à autonomia, a independência, a qualidade de vida e a inclusão de pessoas com deficiência, incapacidades ou mobilidade reduzida.

As autoras ainda destacam que Sartoretto e Bersch (2014) observam que as salas de recursos constituem-se em espaços privilegiados para que o estudante com deficiência aprenda a usar a Tecnologia Assistiva como ferramenta para o desenvolvimento da aprendizagem e da autonomia (NERES; CORRÊA, 2015).

Com a Resolução CNE/CEB $n^{\circ}$ 4, de 2009 (BRASIL, 2009) são instituídas as Diretrizes Operacionais para o AEE na Educação Básica, que orienta, no Art. $5^{\circ}$, que o AEE deve ser realizado, prioritariamente, em Sala de Recursos Multifuncionais. Essa opção, por parte do governo federal, induziu nos últimos anos a um modelo único de oferta do AEE no país, contudo o Atendimento Educacional Especializado oferecido através das Salas de Recursos Multifuncionais, apesar de fundamental, na perspectiva da inclusão escolar, esse não deva ser o espaço exclusivo para esse fim como pretende o MEC (CORRÊA, 2012).

No AEE o uso da Tecnologia Assistiva é previsto por meio do Decreto $n^{\circ} 7.611 / 2011$ (BRASIL, 2011). Com base nesse documento, artigo $2^{\circ}$, a educação especial, através dos serviços de apoio especializado, deve eliminar as barreiras que possam impossibilitar o processo de escolarização de estudantes com deficiência, transtornos globais do desenvolvimento e altas habilidades ou superdotação. No parágrafo primeiro do artigo em pauta, o Atendimento Educacional Especializado é compreendido como:

o conjunto de atividades, recursos de acessibilidade e pedagógicos organizados institucional e continuamente, prestado das seguintes formas [...]: I-complementar à formação dos estudantes com deficiência, transtornos globais do desenvolvimento, como apoio permanente e limitado no tempo e na frequência dos estudantes às salas de recursos multifuncionais; ou II-suplementar à formação de estudantes com altas habilidades ou superdotação. (BRASIL, 2011) 
As orientações do MEC apontam que a composição das SRM se organiza por Salas de Recursos Multifuncionais Tipo I e Salas de Recursos Multifuncionais Tipo II. Ambas constituídas por um conjunto de mobiliários e materiais didático-pedagógicos, equipamentos de informática e outros recursos de acessibilidade, que visam a atender alunos público-alvo da educação especial (CORRÊA, 2012).

Por meio da Nota Técnica Secadi/MEC, n 42/2015(BRASIL, 2015a), fica assegurado que o acesso aos serviços e recursos pedagógicos de acessibilidade nas escolas públicas favorece um maior desenvolvimento acadêmico e social do estudante e, consequentemente, o desenvolvimento inclusivo da escola.

Segundo esse documento, e conforme o disposto pela Portaria Secadi/MEC, $\mathrm{n}^{\circ}$ 25/2012, a Diretoria de Políticas de Educação Especial (DPEE) enfatizou que os recursos de Tecnologia Assistiva, através das Salas de Recursos Multifuncionais, têm como finalidade apoiar a organização e oferta do Atendimento Educacional Especializado. Tais recursos pedagógicos de acessibilidade podem ser utilizados pelo estudante em sala de aula ou em domicílio, não sendo permitida sua utilização para outros fins (BRASIL, 2015a).

E, de acordo com o MEC, através do Programa Implantação de Salas de Recursos Multifuncionais são disponibilizados para estudantes com deficiência os seguintes recursos de Tecnologia Assistiva: mouse com entrada para acionador; mouse estático de esfera; acionador de pressão; teclado expandido com colmeia; lupa eletrônica; notebook com diversas aplicações de acessibilidade; software para comunicação aumentativa e alternativa; esquema corporal; sacolão criativo; quebra cabeça superpostos - sequência lógica; caixa com material dourado; tapete alfabético encaixado; dominó de associação de ideias; memória de numerais; alfabeto móvel e sílabas; caixa de números em tipo ampliado e em braille; kit de lupas manuais; alfabeto braille; dominó tátil; memória tátil de desenho geométrico; plano inclinado; bolas com guizo; scanner com voz; máquina de escrever em braille; globo terrestre tátil; calculadora sonora; kit de desenho geométrico; regletes de mesa; punções; soroban; guias de assinatura; caixa de números em tipo ampliado e em Braille (BRASIL, 2015b, p.67-68).

O que se observa é a diversidade de recursos disponibilizados pelo programa para as Salas de Recursos Multifuncionais no país. Contudo, cabe questionar: de que forma 
esses recursos são disponibilizados no sentido de atender a real demanda dos estudantes que necessitam da oferta de AEE?

Em seus estudos, Verussa (2009) e Rodrigues (2013) evidenciaram que os recursos de Tecnologia Assistiva destinados a estudantes com deficiência, em sua maioria, não estão disponíveis na escola.

$\mathrm{Na}$ área da TA, a Comunicação Aumentativa e Alternativa (CAA) possibilita a construção de novos canais de comunicação, ao valorizar todas as formas expressivas já existentes para pessoas que apresentam dificuldade na escrita ou leitura, ao que Pelosi nos esclarece que:

A Tecnologia Assistiva envolve áreas como: a Comunicação Alternativa e Ampliada; a mobilidade alternativa; o posicionamento adequado; o acesso ao computador; a acessibilidade de ambientes e as adaptações às atividades de vida diária, ao transporte, aos equipamentos de lazer e aos recursos pedagógicos. (PELOSI, 2008 p. 50)

Assim, através de software específico de comunicação alternativa é possível construir pranchas de comunicação que podem ser utilizadas no próprio computador com a função de um vocalizador ou em vocalizadores específicos.

Para os estudantes que apresentam alterações motoras e que dificultam a utilização do mouse convencional, existem os modelos alternativos como joystick, mouse de membrana ou de esfera e também, dispositivos apontadores que direcionam o cursor do mouse seguindo o movimento da cabeça ou dos olhos. A mensagem a ser falada poderá ser feita também por varredura, nesse modo de acesso, um sinal visual ou auditivo seleciona automaticamente os símbolos e o usuário realiza a escolha ativando uma chave acionadora que é colocada em qualquer parte do corpo onde ele tenha controle. Na CAA, existe também o teclado virtual, em que as teclas de letras, de números e demais sinais ficam visíveis no monitor e são selecionadas, uma a uma, produzindo a escrita e a voz (BRASIL, 2010a).

Todavia, Miranda e Galvão Filho (2012) afirmam que ainda existem barreiras que prejudicam o desenvolvimento dessas tecnologias, seja pela falta de conhecimento, seja pela falta de recursos tecnológicos no que diz respeito à legislação ou na própria 
sociedade, através de sua organização de forma a ignorar as diferentes demandas que permeiam a sua população.

No tocante à formação de professores para o uso de Tecnologia Assistiva, em recente atualização dos variados indicadores para a implementação da PNEEI, a Diretoria de Políticas de Educação Especial/Secadi informou que através da Rede Nacional de Formação Continuada de Profissionais do Magistério da Educação Básica Pública, por meio do Programa de Formação Continuada de Professores na Educação Especial, tem entre os cursos oferecidos em nível de aperfeiçoamento, o uso pedagógico dos recursos de Tecnologia Assistiva (BRASIL, 2015a).

Na perspectiva da inclusão escolar, é inquestionável o uso de variados recursos para atender as dificuldades funcionais de estudantes com deficiência, quando os recursos de Tecnologia Assistiva tornam-se importantes. Tais recursos podem ser de alta ou de baixa tecnologia.

Os recursos de baixa tecnologia são aqueles que podem ser construídos pelo professor do AEE e serem utilizados pelo estudante na sala comum ou em outros locais de acordo com a sua necessidade. Já os recursos de alta tecnologia são os adquiridos após a avaliação das necessidades do estudante, sob a indicação do professor de AEE (BRASIL, 2010a).

Verussa (2009, p. 22) traz em seu estudo alguns exemplos e definições a respeito da alta e da baixa tecnologia:

A baixa tecnologia se define por serem de baixo custo, simples e fáceis de fazer. A alta tecnologia se refere aos dispositivos que são caros, mais difíceis de serem construídos e mais difícil de serem obtidos. De acordo com essa distinção, os exemplos de dispositivos de baixa tecnologia vão desde um simples lápis adaptado, livros e utensílios de uso diários adaptados. As cadeiras de rodas motorizadas e os aparelhos de comunicações eletrônicos são exemplos de alta tecnologia. 
Para Galvão Filho (2013), a Tecnologia Assistiva, enquanto um tipo de mediação instrumental ${ }^{4}$, está relacionada com os processos que favorecem, compensam, potencializam ou auxiliam as habilidades ou funções pessoais comprometidas pela deficiência, geralmente relacionadas às funções: motoras, visuais, auditivas e/ou de comunicação.

Ampliando esse entendimento, Pedro (2012, p.88) reitera que as estratégias de ensino de qualquer recurso pedagógico são fundamentais para estudantes com deficiência ou não:

A escolha do software educativo e o planejamento das atividades são importantes e precisam estar associados a estratégias mediadas pelo professor. As estratégias de ensino, as quais possibilitam aos alunos a compreensão e a execução correta das atividades, são imprescindíveis na aplicação de qualquer recurso pedagógico, para alunos com ou sem deficiência.

Os autores em referência, ao assinalarem sobre a importância da diversificação de recursos, indicam que não apenas esses recursos são fundamentais no processo de escolarização de estudantes com ou sem deficiência, como também a atuação dos professores é condição elementar para esse fim, pois potencializam as habilidades desses estudantes rumo ao sucesso escolar, condição vital para a progressão escolar de todos os estudantes na perspectiva da inclusão escolar.

\section{Considerações finais}

Embora o foco deste estudo busque apontar a questão da Tecnologia Assistiva no AEE em SRM para estudantes com deficiência, é digno de nota observar que independente das condições apresentadas pelos estudantes fica evidenciado que o uso de variadas estratégias de ensino, sobretudo aquelas que envolvam a Tecnologia Assistiva, pode se tornar o diferencial nas condições de permanência e de sucesso escolar de estudantes com ou sem deficiência no processo de escolarização.

\footnotetext{
4 Para afirmar o papel da Tecnologia Assistiva como um tipo de mediação instrumental, o autor se fundamenta no conceito de mediação instrumental de Vygotsky (1994).
} 
Ao se constatar a existência de uma variedade de recursos na área da Tecnologia Assistiva e de estudos que nos demonstram a importância desses recursos para o públicoalvo da educação especial, entendemos que pesquisas nessa área são de suma importância para identificar não somente problemáticas, mas também êxitos. A escolha de tais recursos na área da TA deve ter relação com estratégias que permitam a melhor adequação no sentido de assegurar o aprendizado do estudante, pois, cada tipo de deficiência exigirá do professor uma orientação diferenciada.

E que os resultados de outros estudos que se debruçam sobre essa temática possam ser disseminados no sentido de subsidiar práticas que favoreçam a inclusão escolar de forma mais ampla.

\section{Referências}

ASSOCIAÇÃO BRASILEIRA DE NORMAS TÉCNICAS. NBR 9050: acessibilidade a edificações, mobiliário, espaços e equipamentos urbanos. Rio de Janeiro, 2004. Disponível em: < http://www.pessoacomdeficiencia.gov.br/app/sites/default/files/arquivos/\%5Bfield_generi co_imagens-filefield-description\%5D_24.pdf>. Acesso em: 23 jun. 2015.

BERSCH, Rita. Introdução à tecnologia assistiva. Porto Alegre, 2013. Disponível em: < http://www.assistiva.com.br/Introducao_Tecnologia_Assistiva.pdf $>$. Acesso em: 23 jun. 2015.

BRASIL. Comitê de Ajudas Técnicas, Secretaria Especial dos Direitos Humanos da Presidência da República (CORDE/SEDH/PR). Ata da Reunião VII, de dezembro de 2007. Brasília, 2007a. Disponível em: <http://www.mj.gov.br/corde/arquivos/doc/Ata_VII_ Reunião_do_Comite_de_Ajudas_Técnicas.doc>. Acesso em: 30 abr. 2014. 
BRASIL. INSTITUTO NACIONAL DE ESTUDOS E PESQUISA EDUCACIONAIS ANÍSIO TEIXEIRA. Sistema de Consulta a Matrícula do Censo Escolar. Brasília: 2014. Disponível em:

<http://portal.inep.gov.br/basica-censo-escolar-matricula>. Acesso em: 27 out.2015.

BRASIL. Presidência da República. Decreto $n^{\circ}$ 7.611, de 17 de novembro de 2011. Brasília, 2011. Disponível em: <http://www.planalto.gov.br/ccivil_03/_ato2011-

2014/2011/decreto/d7611.htm>. Acesso em: 23 jun. 2015.

BRASIL. Presidência da República. Portaria Normativa n. 13, de 24 de abril de 2007. Brasília, 2007b.

BRASIL. Presidência da República. Lei nº 10.753, de 30 de outubro de 2003. Brasília, 2003. Disponível em:

<http://portal.mec.gov.br/index.php?option=com_docman\&view=download\&alias=9957lei-10753-2003-secadi\&category_slug=fevereiro-2012-pdf\&Itemid=30192 >. Acesso em: 11 set.2014.

BRASIL. Presidência da República. Lei n 9.394, de 20 de dezembro de 1996. Brasília, 1996. Disponível em:<www.planalto.gov.br/ccivil_03/leis/l9394.htm>. Acesso em: 13 set.2014.

BRASIL. Presidência da República. Portaria n 522, de 9 de abril de 1997. Local, data. Disponível em: <http://www.dominiopublico.gov.br/download/texto/me001167.pdf>. Acesso em: 23 jun. 2015.

BRASIL. Ministério da Educação. Secretaria de Educação Especial. Edital n. 01 de 26 de abril de 2007. Programa de Implantação de Salas de Recursos Multifuncionais. Brasília, 2007c.

BRASIL. Ministério da Educação. Secretaria de Educação Especial. A Educação Especial na Perspectiva da Inclusão Escolar. Recursos pedagógicos acessíveis e comunicação aumentativa e alternativa. Brasília: 2010a. Disponível em: <http://ramec.mec.gov.br/seesp/1831-fasc-06-recursos-pedagogicos-acessiveis-ecomunicacao-aumentativa-e-alternativa/file>. Acesso em: 07 jun. 2015.

BRASIL. Ministério da Educação. Nota Técnica n 42/2015/ MEC / SECADI /DPEE. Orientação aos Sistemas de Ensino quanto à destinação dos materiais e equipamentos disponibilizados por meio do Programa Implantação de Salas de Recursos Multifuncionais. Brasília, 2015a. Disponível em: <http://portal.mec.gov.br/index.php?option=com_docman\&view=download\&alias=17656secadi-nt42-orientacoes-aos-sistemas-de-ensino-sobre-destinacao-dos-itenssrm\&category_slug=junho-2015-pdf\&ltemid=30192>. Acesso em: 29 out.2015.

BRASIL. Ministério da Educação. Orientações para implementação da política de educação especial na perspectiva da educação inclusiva. Brasília, 2015b. Disponível em: http://portal.mec.gov.br/index.php?option=com_docman\&view=download\&alias=17237- 
secadi-documento-subsidiario-2015\&category_slug=marco-2015-pdf\&Itemid=30192. Acesso em: 25 maio 2016.

BRASIL. Ministério da Educação. Secretaria de Educação Especial. Política nacional da educação especial na perspectiva da educação inclusiva. Brasília, 2008. Disponível em:< http://portal.mec.gov.br/seesp/arquivos/pdf/politica.pdf >. Acesso em: 13 set.2014.

BRASIL. Conselho Nacional de Educação. Resolução do CNE/CEB nº 4, de 2 de Outubro de 2009. Institui Diretrizes Operacionais para o Atendimento Educacional Especializado na Educação Básica, modalidade Educação Especial. Brasília, 2009. Disponível em: <http://portal.mec.gov.br/dmdocuments/rcebo04_09.pdf>. Acesso em: 28 ago. 2015.

CORRÊA, Nesdete Mesquita. Salas de recursos multifuncionais e plano de ações articuladas em Campo Grande - MS: análise dos indicadores. 2012. 249 p. Tese (Doutorado em Educação) - Universidade Federal de Mato Grosso do Sul, UFMS. Campo Grande, MS, 2012.

GALVÃO FILHO, Teófilo Alves. A construção do conceito de tecnologia assistiva: Alguns Novos Interrogantes e Desafios. 2013. Disponível em:

<http://saci.org.br/index.php?modulo=akemi\&parametro=37809>. Acesso em: 23 Nov.2015.

GALVÃO FILHO, Teófilo Alves. Tecnologia assistiva: favorecendo o desenvolvimento e a aprendizagem em contextos educacionais inclusivos. In: GIROTO, Claudia Regina Mosca; POKER, Rosimar Bortolini; OMOTE, Sadao (Orgs.). As tecnologias nas práticas pedagógicas inclusivas. Marília: Oficina Universitária; São Paulo: Cultura Acadêmica, 2012.p.65-92. Disponível em:<www.marilia.unesp.br/Home/Publicacoes/as-tecnologiasnas-praticas_e-book.pdf >. Acesso em: 09 set.2014.

KASSAR, Mônica de Carvalho Magalhães. Educação especial no Brasil: desigualdade e desafios no reconhecimento da diversidade. Educação e Sociedade, Campinas, v. 33, n. 120, p. 833-849, jul./set. 2012.

MIRANDA, Theresinha Guimarães; GALVÃO FILHO, Teófilo Alves (Org.). O professor e a educação inclusiva: formação, práticas e lugares. Salvador: EDUFBA, 2012. 491 p.

Disponível em:< http://www.galvaofilho.net/noticias/baixar_livro.htm>. Acesso em: 23 jun. 2015.

NERES, Celi Corrêa; CORRÊA, Nesdete Mesquita. Tecnologias assistivas no processo de escolarização de alunos com deficiência sensorial. Revista Educação e Fronteiras On-Line, v. 5, p. 250-262, 2015.

PEDRO, Ketilin Mayra. Softwares educativos para alunos com deficiência intelectual: planejamento e utilização. 2012, 120 p. Dissertação (Mestrado em Educação) -

Universidade Estadual Paulista Júlio de Mesquita Filho, Faculdade de Filosofia e Ciências, Marília, 2012. Disponível em: 
<http://repositorio.unesp.br/bitstream/handle/11449/91196/pedro_km_me_mar.pdf?seque nce=1>. Acesso em: 20 abr. 2016.

PELOSI, Miryam Bonadiu. Inclusão e tecnologia assistiva. Rio de Janeiro, 2008. 303f. Tese (Doutorado em Educação) - Universidade do Estado do Rio de Janeiro, Centro de Educação e Humanidades. Faculdade de Educação. Rio de Janeiro, 2008. Disponível em: <http://www.lateca-uerj.net/publicacoes/docs/Inclus\%C3\%A30\%20e\%20TA\%20-

\%20Tese\%20\%20-\%20Miryam\%20Bonadiu\%20Pelosi\%20-\%202008.pdf> Acesso em: 04 mai. 2016.

QEDU. Censo escolar. Disponível em: <http://www.qedu.org.br/>. Acesso em: 30 jul. 2015.

RODRIGUES, Leda Maria Borges da Cunha. Tecnologia assistiva no processo de inclusão da pessoa com deficiência na rede pública de ensino. 2013. 125 f. Dissertação (Mestrado em Psicologia do Desenvolvimento e Aprendizagem) - Universidade Estadual Paulista Júlio de Mesquita Filho, Faculdade de Ciências, Bauru, 2013. Disponível em:< http://hdl.handle.net/11449/97512>. Acesso em: 25 Nov.2015.

SARTORETO, Mara Lúcia; BERSCH, Rita. Tecnologia assistiva e educação. 2014. Disponível em: <http://www.assistiva.com.br/tassistiva.html>. Acesso em: 3 abr. 2014.

VERUSSA, Edna de Oliveira. Tecnologia assistiva para o ensino de alunos com deficiência: um estudo com professores do ensino fundamental. 2009. 96f. Dissertação (Mestrado em Educação) - Universidade Estadual Paulista Júlio de Mesquita Filho, Faculdade de Filosofia e Ciências, Marília, 2009. Disponível em:

<https://www.marilia.unesp.br/Home/PosGraduacao/Educacao/Dissertacoes/verussa_eo_ me_mar.pdf $>$. Acesso em: 28 out.2015.

VYGOTSKY, Lev Semenovich. A formação social da mente. 5. ed. São Paulo: Martins Fontes, 1994. 